\title{
Tangence
}

\section{Le voyage de la Reine d'Espagne : une nouvelle comédie galante?}

\section{Le voyage de la Reine d'Espagne: a new comédie galante?}

\section{Roxanne Roy}

Numéro 96, été 2011

Le théâtre de la nouvelle : de la Renaissance aux Lumières

URI : https://id.erudit.org/iderudit/1008850ar

DOI : https://doi.org/10.7202/1008850ar

Aller au sommaire du numéro

\section{Éditeur(s)}

Tangence

ISSN

1189-4563 (imprimé)

1710-0305 (numérique)

Découvrir la revue

Citer cet article

Roy, R. (2011). Le voyage de la Reine d'Espagne : une nouvelle comédie galante ? Tangence, (96), 27-49. https://doi.org/10.7202/1008850ar

\section{Résumé de l'article}

Le voyage de la Reine d'Espagne de Jean de Préchac est une oeuvre marquée du sceau de l'hybridité générique. Cette nouvelle galante, qui prend la forme d'une relation de voyage et qui insère des genres mineurs tels que la promenade, les lettres et les billets galants, doit également beaucoup au genre théâtral, puisque plusieurs scènes se présentent comme de petites comédies à lire. Cet article entend mettre au jour la dimension dramaturgique de cette nouvelle en montrant comment Préchac, tout en s'inspirant du Cid, tragicomédie de Corneille, réécrit l'intrigue sur un mode plus comique et galant. Les effets d'ironie dramatique qui en résultent procèdent de la caractérisation des personnages, de la reprise des situations traditionnelles de la comédie ainsi que des procédés comiques d'usage. Enfin, le recours aux thèmes du masque et du déguisement, de même que les mentions relatives aux jeux scéniques et l'ajout de dialogues confèrent à la nouvelle sa théâtralité. Combiner les procédés de l'écriture dramatique et romanesque serait donc une stratégie de Préchac pour diversifier la forme de la nouvelle, genre qui est au goût du jour, et amuser son public friand de nouveauté. 


\title{
Le voyage de la Reine d'Espagne: une nouvelle comédie galante?
}

\author{
Roxanne Roy, \\ Université du Québec à Rimouski
}

Le voyage de la Reine d'Espagne de Jean de Préchac est une œuvre marquée du sceau de l'hybridité générique. Cette nouvelle galante, qui prend la forme d'une relation de voyage et qui insère des genres mineurs tels que la promenade, les lettres et les billets galants, doit également beaucoup au genre théâtral, puisque plusieurs scènes se présentent comme de petites comédies à lire. Cet article entend mettre au jour la dimension dramaturgique de cette nouvelle en montrant comment Préchac, tout en s'inspirant du Cid, tragicomédie de Corneille, réécrit l'intrigue sur un mode plus comique et galant. Les effets d'ironie dramatique qui en résultent procèdent de la caractérisation des personnages, de la reprise des situations traditionnelles de la comédie ainsi que des procédés comiques d'usage. Enfin, le recours aux thèmes du masque et du déguisement, de même que les mentions relatives aux jeux scéniques et l'ajout de dialogues confèrent à la nouvelle sa théâtralité. Combiner les procédés de l'écriture dramatique et romanesque serait donc une stratégie de Préchac pour diversifier la forme de la nouvelle, genre qui est au goût du jour, et amuser son public friand de nouveauté.

$\mathrm{Au} \mathrm{XVII}{ }^{\mathrm{e}}$ siècle, la nouvelle se distingue du roman par sa brièveté et sa concision ${ }^{1}$, par la simplicité et le naturel de son style qui sont garants de la vérité du sujet, par les sujets traités qui sont le plus souvent français, par la condition des personnages qui est plus

1. La brièveté de la nouvelle est un concept assez élastique puisque la nouvelle peut ne compter que quelques pages (comme c'est la cas des histoires qui paraissent dans le Mercure Galant), ou alors avoir plus de 500 pages, la moyenne se situant autour de 250 pages en format du temps (in-12 ${ }^{\circ}$ ). Sur la signification de cette réduction de la nouvelle, lire Nathalie Grande, «Du long au court: réduction de la longueur et invention des formes narratives, l'exemple de Madeleine de Scudéry", XVII siècle, avril-juin 2002, $\mathrm{n}^{\circ} 215$, p. 263-271. 
proche de celle des lecteurs et par les noms qui sont francisés ${ }^{2}$. Tous ces critères ne cherchent qu'à faciliter l'identification du lecteur aux personnages, aux situations et aux milieux où ils évoluent en lui offrant «une image familière de sa propre condition et [en] touch[ant] davantage sa sensibilité ${ }^{3} »$. La poétique de la nouvelle, selon les nouvellistes du temps, s'élabore en tenant compte de deux éléments distincts. D'abord, elle cherche à tout prix à s'affranchir du roman, de sa vraisemblance et de sa fiction, pour privilégier la vérité de l'histoire et se donner un caractère véritable. Ensuite, ses caractéristiques visent surtout à obtenir l'adhésion du lecteur et à plaire à ce public à la recherche constante de nouveaux divertissements. Ce souci du lecteur qui est au fondement de la poétique de la nouvelle répond bien entendu à des enjeux économiques (vendre le plus possible d'exemplaires à un public impatient de les lire), mais il tient aussi du fait que la nouvelle prétend agir sur son public et lui inculquer quelques leçons de morale et de savoir-vivre, car elle se veut un instrument de civilisation. Les nouvelles étant lues principalement par un public d'honnêtes femmes et de mondains ${ }^{4}$, c'est du moins ce qu'affirment Préchac ${ }^{5}$ et

2. Ce paragraphe résume l'essentiel des idées présentées au chapitre 1.1 de mon ouvrage L'art de s'emporter. Colère et vengeance dans les nouvelles françaises (1661-1690), Tübingen, Biblio 17, 2007, p. 26-60.

3. Marie-Thérèse Hipp, Mythes et réalités. Enquêtes sur le roman et les mémoires (1660-1700), Paris, Klincksieck, 1976, p. 44.

4. Ce sont d'ailleurs sensiblement les mêmes lecteurs que ceux qui lisaient des romans, et dont Sorel nous a laissé ce portrait : «Il faut considerer quelles personnes ce sont qui prisent le plus les Romans; on verra que ce sont les Femmes et les Filles, et les Hommes de la Cour et du Monde, soit qu'ils soient gens d'épée, ou que leur oiseveté les fasse plaire aux vanitez du Siecle ", Charles Sorel, De la connoissance des bons livres [1671], Rome, Bulzoni, 1974, p. 133-136. Voir également l'article de Monique Vincent qui présente les lectrices du Mercure Galant où paraissent de nombreuses nouvelles : "Le Mercure Galant et son public féminin ", Romanistische Zeitschrift für Literaturgeischichte, fasc. 1-2, 1979, p. 76-85. Didier Souiller s'interroge sur le public prioritairement féminin des nouvelles dans son chapitre IV: «La nouvelle: une littérature pour les femmes?», La nouvelle en Europe de Boccace à Sade, Paris, Presses universitaires de France, 2004, p. 197-256.

5. «Et comme c'est le beau Sexe qui decide ordinairement de ces petits Ouvrages, j'ay sujet de croire qu'avec la protection du Seigneur de la Cour le plus galand, et de la meilleure mine, qui est le mieux avec les Dames, on aura du moins de l'indulgence pour ma petite Histoire, puisque je ne l'ay écrite que pour vous divertir", Jean de Préchac, L’héroïne mousquetaire, histoire véritable, Paris, Theodore Girard, 1677, p. n. ch. Cette dédicace fait appel à une forme de protectorat qui garantira le succès de l'œuvre mais s'inscrit aussi au cœur d'une vaste entreprise de séduction, ainsi que le remarque Delphine Denis: «elle [l'instance féminine] représente à l'évidence le paradigme d'un 
Valincour ${ }^{6}$, les nouvellistes mettent idéalement en scène des personnages qui, tout comme leurs lecteurs, font preuve d'une certaine bienséance dans leurs comportements et leurs manières, d'un raffinement du langage et de l'expression, d'une maîtrise de leurs passions et de leurs sentiments. Les textes sont marqués d'une élégance stylistique, d'une recherche au niveau des aventures qui sont agréables et galantes, et d'une distinction par le goût. Cet art de plaire qui repose en grande partie sur la scénographie galante ${ }^{7}$ devient donc pour les nouvellistes le meilleur moyen de parvenir à la réussite littéraire et éditoriale, voire financière. Le cas le plus exemplaire est certainement celui de Jean de Préchac qui avoue, à la toute fin de La noble Vénitienne ou la Bassette, histoire galante: "L'auteur ayant perdu quelque argent à la bassette, a trouvé moyen de se dédommager en faisant un livre sur la bassette, dont il a retiré la meilleure partie de ce qu'il avait perdu ${ }^{8}$.»

Fait rare au XVII ${ }^{\mathrm{e}}$ siècle, Jean de Préchac est un écrivain qui vit de sa plume ${ }^{9}$, envisageant l'écriture comme un levier de réussite

lectorat mondain, moderne et citadin, dont elle assume les reproches inégalement fondés d'ignorance, de "naïveté" [...] et de frivolité.» (Le Parnasse galant. Institution d'une catégorie littéraire au XVII siècle, Paris, Honoré Champion, 2001, p. 312).

6. «Croyez-moi, mon cher Monsieur, vous êtes un juge trop sévère, et trop savant pour elle. Ces sortes de bagatelles ne sont point de votre compétence; il faut les renvoyer aux dames et aux cavaliers, qui en jugeront mieux que vous » (Valincour, Lettres à Madame la Marquise ${ }^{* * *}$ sur la Princesse de Clèves [1678], Paris, Garnier-Flammarion, 2001, p. 64).

7. Rappelons qu'au XVII siècle, l'esthétique de la galanterie, cet art de plaire avec enjouement qui valorise la nouveauté, la délicatesse et les grâces légères, est indissociable d'un certain mode de vie mondain. En effet, la notion de galanterie tient tout autant d'une pratique sociale (celle des salons) que d'une pratique littéraire. Elle définit les manières de se conduire dans le monde, d'aimer et d'écrire, la finesse du goût, du langage, de l'esprit et des sentiments. Sur les ambiguïtés du terme galant, on consultera Alain Viala, «La littérature galante: histoire et problématique", Il Seicento francese oggi. Situazione e prospettive della ricerca, Bari et Paris, Adriatica et Nizet, 1994, p. 100-113. Pour une théorisation de la galanterie, voir Delphine Denis, Le Parnasse galant, ouvr. cité, p. $95-123$.

8. Jean de Préchac, La noble Vénitienne ou la Bassette, histoire galante, Paris, Compagnie des Libraires associés, 1678, p. 186.

9. Jean de Préchac est un «romancier courtisan», pour reprendre les termes de Jacques Chupeau dans son excellent article «Jean de Préchac, ou le romancier courtisan ", dans Jean Serroy, Romanciers du XVII siècle, Paris, Klincksieck, 1991, p. 271-289. Né en 1647 à Buzy, Préchac est reçu avocat au Parlement de Navarre en 1669, puis il occupe le poste de lecteur de Monsieur en 1676, ainsi que celui de secrétaire et professeur d'espagnol de Marie-Louise d'Orléans 
destiné à lui procurer une certaine reconnaissance et à assurer sa réputation. Pour ce faire, il entend bien tirer parti de la curiosité d'un public mondain friand d'historiettes et de nouvelles: à charge pour lui de composer des histoires galantes toujours étonnantes et divertissantes, en explorant diverses formes d'écriture. Cette volonté de se renouveler sans cesse est manifeste dans le cas des nouvelles qu'il présente sous la forme d'une relation de voyage ${ }^{10}$. En effet, il s'inspire de ce genre pour varier les procédés narratifs en écrivant le récit des aventures singulières et des intrigues amoureuses qui se sont déroulées au cours d'un voyage, mais ce choix n'est pas fortuit, puisqu'il correspond à la vogue des récits de voyage qui a cours alors. Tout se passe comme si l'auteur voulait profiter de cette mode pour relancer celle de la nouvelle et lui donner ainsi un nouveau lustre.

Le voyage de la Reine d'Espagne, publié à Paris en 1680, est une œuvre marquée du sceau de l'hybridité générique. D’abord, ainsi que le précise le sous-titre, il s'agit d'une nouvelle galante, c'està-dire un texte narratif relativement court inspiré de l'actualité qui raconte les intrigues amoureuses (le plus souvent malheureuses) d'un individu particulier. Ce type de nouvelle se caractérise notamment par des repères spatio-temporels entourés d'un certain flou et par un récit qui insiste sur les passages galants (conversations,

jusqu'au mariage de celle-ci en 1679. Après 1690, il s'éloigne de la cour et devient conseiller au Parlement de Navarre (en mai 1693); il obtient la charge de Garde-Scel de la chancellerie de Pau en mars 1696. Il meurt le 12 mars 1720 à Pau. Auteur prolifique, Jean de Préchac fait paraître entre 1677 et 1698 une trentaine d'ouvrages de fiction qui ont remporté un franc succès. C'est le cas notamment de L'héroïne mousquetaire, histoire véritable (1677) : rééditée trois fois en France (1681, 1713 et 1722), la nouvelle a donné lieu à quatre éditions hollandaises et elle fut traduite et rééditée en anglais de même qu'en flamand. Sur le rayonnement et la réception des œuvres de Préchac, voir l'article de Rudolf Harneit, «Réception de $\mathrm{M}^{\text {me }}$ de Villedieu et Préchac en Europe», dans Nathalie Grande et Edwige Keller-Rahbé (dir.), Littératures classiques, $\mathrm{n}^{\circ} 61$ (Madame de Villedieu ou les audaces du roman), printemps 2007, p. 275293. Sur la vie et les œuvres de Préchac, on consultera avec profit l'introduction de Françoise Gevrey dans Jean de Préchac, Contes moins contes que les autres précédés de L'Illustre Parisienne, Paris, Société des Textes Français Modernes, 1993, p. i-xlix.

10. Jean de Préchac, Le voyage de Fontaine-bleau, Paris, La compagnie des marchands libraires associez, 1678 ; Jean de Préchac, Le voyage de la Reine d'Espagne, Paris, Jean Ribou, 1680, 2 vol.; Jean de Préchac, Relation d'un voyage fait en Provence, contenant les antiquitez les plus curieuses de chaque ville, et plusieurs histoires galantes, Paris, Claude Barbin, 1683; Jean de Préchac, Le fameux voyageur, Paris, Padeloup, 1682. 
rencontres furtives, bals, lettres et cadeaux échangés, rêveries, dilemmes amoureux, etc.) afin de séduire le lecteur ${ }^{11}$. Dans la nouvelle de Préchac, on suit donc les amours sans cesse entravées de Perline et du comte de Beaujeu. Mettant à profit la mode des relations de voyage, Préchac donne pour cadre à son intrigue un événement historique récent, bien connu du lecteur. En quelques pages — trois tout au plus —, il évoque le mariage de Charles II, roi d'Espagne, et de Marie-Louise d'Orléans, fille du duc d'Orléans, puis il décrit le cortège et les circonstances du voyage entrepris par celle-ci pour retrouver son époux à Burgos. Les réjouissances qui marquent chacune des étapes du voyage de la reine, tout comme les incommodités liées à ces déplacements - depuis Paris, Poitiers, Blayes, Bordeaux, en passant par Castres, Bazas, Mont-de-Marsan, Dax jusqu'à Burgos - ponctuent l'intrigue amoureuse, favorisant tantôt la rencontre des amants, étant tantôt à l'origine d'une douloureuse séparation. La toile de fond historique devient surtout un prétexte pour écrire une nouvelle galante et authentifier la narration. Préchac ayant lui-même pris part au voyage, il se présente comme un témoin de cette "fidelle histoire ${ }^{12}$ » et souligne du coup son caractère véridique. Il est à remarquer que l'auteur s'inspire de préférence d'une forme narrative proche de celle qu'emprunte le récit historique, donc plus près de faits avérés, afin de renforcer la crédibilité de sa nouvelle et d'échapper à une critique prompte à condamner l'irréalisme qu'on attribue souvent au roman.

La forme de la promenade, proche parente de la relation de voyage, est également présente lors de quelques scènes-clés de la nouvelle. Comme la promenade raconte une histoire située dans

11. La nouvelle galante se distingue en cela de la «nouvelle de ce temps» qui rapporte des aventures contemporaines qui se sont récemment déroulées dans le milieu de la cour, et de la "nouvelle historique» dont l'intrigue se situe dans un cadre historique, un temps et des lieux précis. Elle met en scène des personnages publics qui ont fortement marqué leur époque et elle rapporte des faits réels et vérifiables (guerres, batailles, troubles politiques, coups d'état, etc.). Ce type de nouvelle tend à expliquer les événements politiques par l'analyse des mobiles secrets et des motivations internes de personnages illustres, où l'amour, l'ambition et le ressentiment jouent souvent un rôle de premier plan.

12. Jean de Préchac, Le voyage de la Reine d'Espagne, ouvr. cité, t. I, p. 11-12. Dans l'épître à son altesse royale mademoiselle, on lit: "Mademoiselle, Le Voyage que j'ay fait avec la Reine d'Espagne, m'ayant donné occasion d'écrire cette petite Histoire», n. p. Désormais, les références à cet ouvrage seront indiquées par le sigle $V R E$, suivi de la page, et placées entre parenthèses dans le corps du texte. Quant au tome II, les références seront indiquées par le sigle VRE II. 
le cadre d'une activité fort prisée par les mondains et dans les endroits même qu'ils fréquentent (Versailles, les Tuileries, etc.), l'auteur espère sans doute par là obtenir l'adhésion du lecteur et lui plaire ${ }^{13}$. Ainsi, dans le cadre du Voyage de la Reine d'Espagne, les jardins d'un château deviennent un lieu galant de prédilection, propice aux rencontres et aux conversations les plus tendres. Préchac, par ailleurs, insère un genre mineur au sein même de sa nouvelle. À onze reprises, on trouve en effet des billets et des lettres mis en retrait dans la nouvelle par un jeu typographique, et qui sont essentiellement au service du commerce amoureux entre Perline et le comte de Beaujeu. Les amants tâchent par ce moyen de communiquer malgré la distance, s'informant du déroulement de leurs affaires et s'assurant mutuellement de leur amour. Or, il nous semble que Le voyage de la Reine d'Espagne doit aussi beaucoup aux genres dramatiques et que plusieurs des épisodes qui ponctuent la nouvelle sont, en fait, de véritables scènes de comédie. Cette théâtralité de l'écriture pourrait bien être une des particularités de la production «nouvellistique» de Préchac et c'est ce que nous entendons mettre au jour ici en analysant le sujet de l'intrigue, la caractérisation des personnages et le topos du déguisement.

\section{La tragicomédie}

Plusieurs indices autorisent ce rapprochement entre le théâtre et Le voyage de la Reine d'Espagne. Premièrement, la dimension dramaturgique de la nouvelle se manifeste par les jeux de renvois intertextuels, puisque, à bien des égards, le sujet du Voyage de la

13. C'est le cas notamment de $\mathrm{M}^{\text {lle }}$ de Scudéry qui ne cesse de chercher sa voie depuis le déclin des longs romans et qui favorise plutôt la nouvelle/promenade (Madeleine de Scudéry, La promenade de Versailles [1669], Genève, Slatkine Reprints, 1979). Cette volonté de plaire au public mondain, dont témoigne à l'évidence l'auteure par le choix des lieux de promenade, n'est pas sans rappeler celle de La Fontaine dans Les amours de Psyché et de Cupidon qui paraissent la même année. Voir aussi l'ouvrage anonyme, La promenade de Livry, Paris, Charles Osmont, 1678, 2 vol. La forme de cette nouvelle est plus complexe, puisque le narrateur écrit à une dame pour lui raconter ce qui se passa dans la compagnie de sept ou huit honnêtes personnes (dames et cavaliers) lorsqu'elles se retirèrent dans la forêt de Livry pour profiter de la douceur de la campagne et échapper aux contraintes de Paris, ce qui donne lieu au récit de six petites histoires. Sur la forme de la "Promenade», lire Delphine Denis, «Du Parterre aux Promenades: une scène pour la littérature du $\mathrm{XVII}^{\mathrm{e}}$ siècle", XVII siècle, $\mathrm{n}^{\circ}$ 209, 2000, p. 655-670, et Alain Montandon, «Des promenades ", Les espaces de civilité, Mont-de-Marsan, Éditions Interuniversitaires, 1995, p. 53-78. 
Reine d'Espagne semble s'inspirer librement de la tragicomédie du Cid de Corneille. Si la pièce n'est jamais directement citée — sans doute en raison d'un succès qui perdure tout au long du siècle plusieurs passages faisant appel à la compétence du lecteur l'invitent à la reconnaître en sous-texte. D’abord, Préchac modifie légèrement l'intrigue, puisque c'est le marquis de Pontignac, le frère de Perline, et non son père, qui est tué par son amant. Puis il la transpose sur un mode résolument comique. Dès le début de la nouvelle, le frère de Perline est présenté comme un personnage brutal, grossier et sans discernement ${ }^{14}$, défauts qui sont encore soulignés lors du combat qui s'engage entre ce dernier et le comte de Beaujeu. Préchac transforme le duel en un combat vain et ridicule, en lui donnant un motif condamnable où l'honneur n'a pas de part. En effet, le marquis de Pontignac croit devoir venger sa mère dont les charmes ont été méprisés par le comte de Beaujeu: «Le jeune Pontignac sans examiner autre chose, crût que son honneur estoit interessé dans cette affaire, et ayant pris ses avantages pour se battre contre Beaujeu, il le rencontra ${ }^{15}$ le mesme jour, et lui fit tirer l'épée, le Comte qui n'avoit qu'une épée fort courte, comme le sont d'ordinaire celles des gens de la Cour, ne songea qu'a se deffendre» (VRE, p. 149-150). L'auteur met de l'avant le manque de noblesse du personnage qui se lance dans un combat à armes inégales où il s'assure d'avoir l'avantage, ce qui rend le marquis et sa cause d'autant plus méprisables. Sa mort triviale n'a rien d'héroïque, puisque le marquis s'empale lui-même sur l'épée de son rival, ce qui accentue encore le ridicule du combat et du personnage. La réaction de ses proches et de son entourage, qui considèrent que le marquis est mort par sa faute, contribue de même à le discréditer complètement et à désamorcer le tragique de la situation: «Dulac aprit en arrivant à Bordeaux, que tous les honnestes gens blâmoient Pontignac, qui s'étoist enferré lui-mesme dans l'épée du Comte, aprés l'avoir attaqué avec avantage» (VRE, p. 160-161). L'auteur incite du coup le lecteur à y voir une scène de

14. Au sujet du jeune marquis de Pontignac, Préchac écrit: «Ce fils qui étoit aussi brutal que sa sœur estoit aimable, faisoit toutes ses delices» (VRE, p. 12); plus loin il le qualifie de «fils fort indigne» (VRE, p. 15); puis il termine ce portrait en précisant qu'il est «fort étourdy, et de fort mauvaise compagnie» (VRE, p. 35).

15. Notons que Préchac prend soin de préciser qu'il s'agit d'une rencontre et non d'un duel, ce qui est de la plus haute importance pour la suite de l'intrigue car cela permet au comte de gagner le procès que la mère de Perline va lui intenter en évoquant les édits du roi contre les duels. Atténuant ainsi la potentielle tension dramatique de l'intrigue, l'auteur peut en faire un ressort comique. 
comédie, le public ayant naturellement tendance à s'identifier aux personnages qui font preuve de bon sens plutôt qu'à celui dont on se moque.

Le dilemme vécu par Perline et le comte de Beaujeu, à la suite du décès du marquis de Pontignac, évoque le conflit cornélien de Chimène et de Rodrigue, où la gloire, l'honneur et la bienséance deviennent autant d'obstacles à leur amour. Si la nouvelle de Préchac conserve certaines similitudes avec la pièce de Corneille, il la réécrit toutefois en donnant préséance à la galanterie et à l'ironie. Dans un premier temps, le nouvelliste situe son intrigue du côté de la tragédie et du pathétique, notamment par le biais du personnage de Perline qui suscite la pitié par son affliction, ses tristes lamentations et ses larmes. Bien que sa douleur face à la mort de son frère soit réelle, son amour pour le comte de Beaujeu est toujours vivant, la livrant ainsi aux plus cruels tourments: «si son amour la flattoit quelquefois de quelque étincelle d'esperance, la raison la détruisoit bien-tost après, en lui représentant qu'elle devoit étouffer ces foibles sentimens, si indignes d'une sœur qui venoit de perdre son frère" (VRE, p. 159). Telle une autre Chimène, Perline ne peut aimer l'assassin de son frère, si bien que les questions de morale et de bienséance sont au cœur du débat qui la déchire: "elle s'en deffendit long-temps, sur ce que la bienseance ne luy permettoit pas d'avoir commerce avec un homme qui venoit de tuer son frère» (VRE II, p. 27). Le péril tragique s'accentue lorsque Perline promet à sa mère mourante de poursuivre sa vengeance contre le comte de Beaujeu. En fait, le discours que la marquise de Pontignac tient à sa fille rappelle celui des grandes tragédies, dans la mesure où le conflit se structure autour des mêmes enjeux: "elle luy dit, qu'elle n'avoit plus de regret à mourir, ne doutant point qu'elle ne fit son devoir pour perdre l'assassin de son frère, puisque sa gloire, son honneur et celuy de sa famille y estoient engagez $»(V R E I I$, p. 82-83).

La promesse faite à sa mère n'est pas sans conséquence, puisqu'elle contraint Perline à jouer la comédie pour ne pas encourir la réprobation générale et le déshonneur. Afin de préserver les apparences, elle feint la fille éplorée, assoiffée de vengeance, et «elle s'avisa mesme, pour mieux tromper le public, d'écrire à son oncle le Président, et de le prier de venir à Paris, pour l'aider de son credit, et de ses conseils » (VRE II, p. 87-88). Le stratagème s'avère efficace, puisque le comte de Beaujeu renonce à sa liberté et s'en remet entièrement à la volonté de sa maîtresse. Son dévouement et 
sa générosité font, au surplus, écho aux vertus de Rodrigue, puisque le comte offre sa tête à sa maîtresse pour satisfaire sa vengeance et lui prouver du même souffle son indéfectible amour: «car je vous declare que j'abandonne le soin de mon affaire, ayant bien moins de peine à me resoudre à mourir, qu'a m'opposer à vos volontez» (VRE II, p. 89-90, en italique dans le texte). Alors que la tension dramatique est à son apogée, Perline dévoile, dans un billet destiné à Beaujeu, ses réelles motivations en des termes faisant appel aux valeurs héroïques et ramenant dès lors l'intrigue sur le terrain de la tragicomédie ${ }^{16}$ :

Pouvez-vous penser, ingrat, que je veüille vostre mort, puisque je ne la poursuis que pour vostre gloire, quel jugement feroit de moy le public, si j'abandonnois les interests de ma maison, un moment aprés la mort de ma mère, et quelle gloire auriés-vous d'épouser une personne, qui auroit trahy son propre sang pour suivre les mouvemens déreglez d'une passion aveugle, contentez vous de sçavoir mes foiblesses (VRE II, p. 91-92, en italique dans le texte).

Au même moment, les interventions du narrateur et les pointes ironiques qui ponctuent le texte contribuent grandement à désamorcer le tragique de la situation: «et puisque la bonne ou la mauvaise conduite d'une fille ne consiste que dans l'opinion que le monde en a, ne souffrez pas que je me deshonore en faisant connoistre nostre intelligence» (VRE II, p. 92, en italique dans le texte). Cette remarque piquante sur une morale curiale qui ne se soucie que des apparences reprend le topos de la cour qui se présente tel un vaste théâtre du monde où chacun tient un rôle, où chacun se surveille et s'épie ${ }^{17}$. C'est précisément le poids du regard des autres qui contraint Perline à jouer cette comédie contre son gré, ainsi qu'on peut le lire dans cet extrait: "plaignez la bizarrerie de ma destinée, qui m’engage à demander ce que je

16. Un précédent billet de Perline, destiné au comte de Beaujeu, préparait déjà le lecteur à ce revirement de situation: "souvenez-vous que la bien-seance sera toûjours une foible raison pour m'empescher de vous voir, lors qu'il ne dépendra plus que de moy» (VRE II, p. 75, en italique dans le texte).

17. Cette conduite dictée par les contraintes et les réseaux d'interdépendances, qui exercent des pressions de plus en plus fortes sur les individus évoluant dans cette hiérarchie sociale déterminée qu'est la société de cour, ainsi que l'a montré Norbert Élias (La société de cour [1974], Paris, Flammarion, 1985), indique clairement que l'art d'observer ses semblables et de contrôler ses passions, donc l'art de connaître les hommes, est un art crucial dans l'univers de la cour. Le courtisan, fort de ce savoir, épie sans cesse son voisin ou son ennemi, et l'art de déchiffrer un visage devient une arme essentielle pour qui veut élucider les intrigues de la cour. 
serois au desespoir d'obtenir» (VRE II, p. 92-93). D'ailleurs, l'auteur souligne clairement à quel point il est difficile pour Perline de tenir en public le rôle de la jeune fille qui veut à tout prix obtenir la tête du comte de Beaujeu, ce qui l'incite à fuir dans un couvent pour se mettre à l'abri des regards indiscrets: "Perline qui n'avoit plus la force de soustenir un caractere si opposé à ses veritables sentimens, $[\ldots]$ se retira dans une maison Religieuse, sur le pretexte d'y aller faire des exercices de pieté pendant le Caresme » (VRE II, p. 95-96). Le lecteur, tout comme le personnage du comte de Beaujeu, connaissant les véritables motivations de Perline, n'a plus à craindre son funeste désir de vengeance. Rassuré sur le sort qui attend Beaujeu — sa vie n'étant pas réellement en péril —, il est alors invité à apprécier le rôle de composition que la jeune fille tient dans cet épisode de la nouvelle et à lire ces scènes comme de véritables comédies.

Le stratagème final, qui vise à persuader Perline de se marier au comte de Beaujeu sans plus attendre, n'est pas sans rappeler la ruse employée par le roi d'Espagne, lorsque ce dernier fait croire à Chimène que Rodrigue est mort pour calmer son inexorable courroux, puis la faire consentir à épouser le Cid. Toutefois, c'est en recourant à un procédé comique, celui de l'équivoque et du comique de mot, que Préchac amorce la scène finale. Considérant le serment qu'a fait Perline à sa mère mourante "de ne se marier jamais qu'à un homme qui luy apportât la teste du Comte de Beaujeu» (VRE II, p. 162-163), ce dernier lui écrit un billet dans lequel il joue sur le double sens des termes: «on m'asseure que vous demandez ma teste, je cours la livrer à la personne qui doit vous la presenter» (VRE II, p. 165, en italique dans le texte). En n'apercevant dans cette déclaration que son sens littéral, Perline se méprend sur l'identité de l'inconnu qu'elle doit rencontrer. Persuadée qu'elle va recevoir la tête du comte de Beaujeu, elle est alors en proie à une douleur et un désespoir sans borne. Le lecteur, informé de cette ruse, est en mesure de rire de la confusion dans laquelle se trouve Perline et d'apprécier le comique de la situation, de même que le comte de Beaujeu, «qui l'attendoit, eut le plaisir d'estre témoin d'une partie de ses emportemens» (VRE II, p. 171). Coup de théâtre, c'est le comte de Beaujeu qui vient s'incliner à ses pieds et Perline s'étant à peine remise de sa surprise "se détermina à ne différer plus son mariage » (VRE II, p. 172-173). Par ce revirement final, la scène passe donc du tragique au comique, ou à la tragédie qui finit bien, puisque le mariage entre les amants est enfin célébré. 
On le voit, Préchac n'hésite pas à tirer profit du succès d'une œuvre, en l'occurrence Le Cid, pour composer sa nouvelle, espérant par cette stratégie plaire au public. Ce faisant, il puise dans le répertoire dramatique de son temps une pièce dont l'appartenance générique ambiguë lui permet de jouer sur un double registre, celui du tragicomique. Toutefois, à la lecture, on constate qu'il évite constamment la tentation du tragique en réécrivant l'intrigue sur un mode résolument comique et galant, tissant délibérément des liens thématiques et formels de plus en plus étroits entre le théâtre et la nouvelle.

\section{Le comique de caractère et l'ironie dramatique}

La caractérisation des personnages dans Le voyage de la Reine d'Espagne pourrait bien être un autre procédé conférant une dimension dramaturgique à la nouvelle de Préchac. Plutôt que de décrire physiquement et moralement ses personnages, l'auteur brosse leur portrait à grands traits, se contentant d'employer des qualificatifs hyperboliques, insistant essentiellement sur la vertu et la beauté des protagonistes, ce qui a pour effet de les rapprocher des types généraux que l'on trouve dans les comédies. Ainsi, Perline est présentée comme "une fille dont la beauté estoit aussi singuliere que ses biens estoient considerables» (VRE, p. 6), «une fille si parfaitement belle» (VRE, p. 7) et " qui avoit cette vivacité si ordinaire aux personnes de son païs» (VRE, p. 25). Quant au «jeune Comte de Beaujeu» (VRE, p. 7), on sait seulement qu'il «se fist distinguer par sa bonne mine» (VRE, p. 9), et que Perline se félicite d'être aimée «d'un Cavalier, qui avoit tous les avantages de l'esprit, de la naissance, et de la bonne mine» (VRE, p. 33-34). Aux côtés des parfaits amants, on trouve les personnages traditionnels de la comédie, qui mettent en péril leur amour et leur projet de mariage: la mère acariâtre et tyrannique et le prétendant incommode qui veut naturellement épouser la jeune promise. Pour mieux souligner leur antagonisme, Préchac insiste sur la laideur physique et morale des personnages secondaires, écrivant au sujet de la marquise de Pontignac qu'elle «estoit la femme du monde qui avoit le plus de vanité, et qui estoit plus sensible aux flateries » (VRE, p. 12) et de l'oncle de Perline qu'il est « un vieux President qui aimoit avec passion cette belle fille» (VRE, p. 13). Ces deux figures, par leurs vices ridicules et condamnables, deviennent, à leur insu, des personnages de comédie dont on se moque et c'est ce que nous voudrions mettre au jour dans les lignes qui suivent. 
Le plus souvent, c'est la passion qui incite les amants à jouer un rôle et à duper les autres personnages pour parvenir à leurs fins, en un mot, à simuler comme on le fait habituellement dans les comédies de l'amour. Dans son étude sur Le Portefeuille de $\mathrm{M}^{\mathrm{me}} \mathrm{de}$ Villedieu, Jean Garapond affirme d'ailleurs qu'il n'y a «[p]as de plaisir plus délectable pour les amants que celui de s'aimer à l'insu de tous, de jouer en public la comédie de l'indifférence, non pour garder pur un sentiment qui se dégrade sous le regard des autres, mais afin de se donner en spectacle à ces mêmes autres ${ }^{18}{ } »$. Ces propos conviennent tout autant à la nouvelle de Préchac, puisqu'on y trouve une situation similaire. D'emblée, on note que l'auteur prépare soigneusement la scène, insistant particulièrement sur le cadre et le costume, afin d'accentuer la théâtralité de la comédie en trois temps qui va se dérouler sous les yeux du lecteur. La première scène se déroule lors d'un bal tenu chez la reine où Perline et sa mère portent toutes deux un masque de bohémienne. Ce déguisement, selon le procédé traditionnel de la comédie, est à l'origine d'une méprise sur l'identité puisque le comte de Beaujeu, «trompé par le masque et par la ressemblance des habillemens» (VRE, p. 41), tient des propos fort tendres à la marquise qu'il prend pour Perline jusqu'au moment où il reconnaît la voix de la mère. Une fois détrompé, le comte décide de retourner la situation à son avantage. Afin de garder secret son amour pour Perline et d'endormir la défiance de la marquise de Pontignac, le comte de Beaujeu feint d'être épris de ses charmes. Son jeu d'acteur est convaincant, puisque la mère de Perline est dupée par cette comédie qui repose partiellement sur le comique de caractère. En effet, sa vanité la rend crédule, facile à berner et du même coup ridicule. Cherchant à se divertir davantage et à faire d'une amante importune un personnage de comédie, selon le procédé de la méprise sur les sentiments, le comte de Beaujeu prétend agréer sa cour. Ses propos sont d'ailleurs explicites quant à ses intentions et au rôle qu'il prétend faire jouer à la mère de Perline: «il voulut voir jusqu'où pourroit aller la folie de cette belle Surannée» (VRE, p. 43). La marquise tient donc un rôle sans s'en rendre compte, ce qui la rend encore plus risible.

Cette maxime piquante du narrateur, qui est loin d'être tendre à l'égard des femmes vieillissantes, donne le ton à la comédie qui

18. Jean Garapond, "Le Portefeuille, roman d'éducation en miniature", dans Nathalie Grande et Edwige Keller-Rahbé (dir.), Littératures classiques, ouvr. cité, p. 138. 
va suivre et précise du même coup le vice qu'il entend condamner moralement: "toutes les femmes se flattent, et [...] l'âge ne les guerit pas toûjours de la bonne opinion qu'elles ont de leur beauté» (VRE, p. 43). Préchac construit son personnage sur un défaut universel et général, qu'il va grossir au point d'en faire une caricature et de transformer la mère de Perline en type même de la coquette vieillissante qui croit pouvoir être aimée d'un jeune et beau cavalier. Ce trait est particulièrement visible au deuxième temps de la comédie lorsque la marquise, qui prétend se présenter telle une jeune amoureuse aux yeux du comte, soigne (en vain) son apparence, maquillant et fardant son visage, puis veillant à bien ajuster ses habits: «elle n'oublia rien pour y reüssir, et n'épargna aucune des pommades de la nouvelle invention pour cacher ses rides» (VRE, p. 47). Ayant invité son prétendu amant à dîner chez elle, elle tient des propos qui soulignent vivement sa vanité et sa coquetterie, ce qui achève le portrait caricatural de cette coquette. Le comique de mot, les antithèses et les paradoxes abondent dans les répliques de la marquise, comme le montre l'extrait suivant: «avoüez, Monsieur, continua-t'elle, qu'une jeune fille a bien des imperfections, et qu'il luy faut bien du temps pour acquérir cét air du monde, et cette noble majesté que les femmes d'un âge plus meur ont d'ordinaire quand elles sont naturellement belles» (VRE, p. 59-60). La marquise ne perçoit pas l'ironie des remarques du comte, qui renchérit à ce sujet et l'incite à développer plus longuement sa pensée pour mieux se divertir de ses propos insensés: «il luy dit mesme, qu'il ne connoissoit point de femme en France, qui eut l'air et les manieres d'une femme de qualité comme elle, et que pour luy, il n'auroit jamais deviné à la voir si jeune, que Pontignac et sa sœur fussent ses enfans» (VRE, p. 60-61). Le lecteur, qui en apprécie toute la portée, est invité à imiter la réaction du comte et de Perline: "Le Comte jettoit de temps en temps les yeux sur Perline, qui ne pouvoit plus s'empescher de rire de la folie de sa mere» (VRE, p. 62). Le comique de geste n'est pas en reste puisque le comportement de la marquise, qui gave son invité plutôt que de le régaler, souligne son manque de finesse et de discernement: «le Comte en auroit esté fort content, si la Marquise ne luy eut servy indifferemment de tout ce qui estoit sur la table, sans luy laisser la liberté de manger, et ne l'eut accablé de complimens, en luy demandant toûjours pardon de ce qu'elle ne luy faisoit pas assez bonne chere»(VRE, p. 62-63). La marquise apporte ainsi un démenti aux propos tenus plus tôt, car son ignorance des usages du monde et des règles de galanterie les plus élémentaires trahit 
son âge et son attitude laisse supposer qu'elle n'a pas eu d'amants depuis longtemps.

Aveuglée par son amour, la marquise ne s'aperçoit pas des tromperies et moqueries dont elle est l'objet, au contraire des autres personnages du Voyage. En effet, à plusieurs reprises, Préchac mentionne la réaction de l'entourage, de plus en plus élargi, qui, ne croyant pas à l'amour prétendu du comte pour la marquise, regarde cette femme comme une coquette qui se donne en spectacle et dont on se rit. D'abord, le comte de Beaujeu et Perline s'amusent de l'erreur dans laquelle ils entretiennent la mère, étant à la fois les acteurs et les spectateurs de cette comédie. Par exemple, sous prétexte de vouloir se ménager un entretien seul à seul avec la marquise, le comte l'incite à se retirer dans sa chambre et, pendant que la mère l'attendait, il «se rendit auprès de Perline, et l'accompagna à la promenade, où il passerent la plus grande partie de l'apresdîner ensemble, ce ne fut pas sans rire de la folie de la Marquise» (VRE, p. 113). Ensuite, le Président, qui se refuse à croire que le jeune comte puisse aimer la marquise, qu'il regarde comme « une femme d'une vanité insupportable» (VRE, p. 79), devine la supercherie et n'entre pas dans la comédie, devenant plutôt un spectateur. Puis, l'entourage de la reine est difficilement berné par les propos de Beaujeu qui avoue aimer la marquise afin de protéger son amour pour Perline: «le Comte, pour leur donner le change, leur dit en particulier qu'il estoit amoureux de la Marquise, il ne trouva personne qui voulut le croire, mais la Marquise avoit tant d'attention à le regarder toutes les fois qu'elle le rencontroit, qu'on commença à juger qu'il ne luy estoit pas indifferent» (VRE, p. 106-107). Dans la nouvelle, la marquise est donc perçue comme un personnage de comédie, et tous, personnages et lecteurs, au fait de la méprise, assistent avec amusement à la scène qui se joue.

Cette comédie prend fin abruptement lorsque le comte de Beaujeu, lassé de ce divertissement, cesse de bien tenir son rôle et rit d'elle publiquement ${ }^{19}$, ce que la marquise finit bientôt par apprendre: "elle jugea que le Comte pouvoit avoir quelque part au ridicule qu'on luy donnoit dans le monde» (VRE, p. 119). Le mépris de ses charmes et la trahison dont elle est victime animent alors sa colère et font naître un insatiable désir de vengeance;

19. «[I]l prenoit si peu soin d'en desabuser le monde, et se deffendoit si mal toutes les fois que ses amis luy faisoient des plaisenteries sur la bonne intelligence où il etoit avec la Marquise, qu'il confirma par cette conduite tous les soubçons qu'on en avoit déja» (VRE, p. 117-118). 
la marquise n'aura de cesse, par la suite, de réclamer la tête de Beaujeu et de nuire aux amours de Perline et du comte. Le comique relève donc ici de l'ironie dramatique selon un mode de fonctionnement qui se déploie en trois temps ${ }^{20}$ : d'abord l'exposition, le personnage (la marquise de Pontignac) ignorant une information (les véritables sentiments du comte de Beaujeu) que connaît le spectateur (le lecteur); ensuite l'exploitation, qui met en scène avec ironie les conséquences de cette situation (l'ignorance où se trouve la marquise la fait tomber dans un piège et les discours qu'elle tient, de même que son entreprise pour séduire le comte, la rendent ridicule); et enfin la résolution, lorsque la marquise découvre que le comte de Beaujeu se moque d'elle et que tout le monde rit de sa folle passion.

L'oncle de Perline est également peint sous les traits d'un ridicule personnage de comédie, en incarnant le rival du comte sous la figure du vieux mari incommode ${ }^{21}$. On le décrit comme un homme dont «la jalousie lui fit tout craindre» (VRE, p. 64), un «fâcheux» (VRE, p. 95) qui porte ombrage au commerce entre les jeunes amants, au point où Perline fuit «les fatiguans discours de [s] on haïssable oncle» (VRE, p. 128). Préchac recourt au comique de caractère, jouant sur l'obsession ridicule du vieil oncle et tournant en dérision ce personnage entêté qui veut se marier avec Perline sans se soucier du bon sens et sans vouloir entendre raison. D'abord, Préchac précise que le Président aime en dépit de la morale et de la loi, condamnant du coup sa folle passion. Perline étant une trop proche parente, il doit obtenir une dispense de Rome pour l'épouser: "Cependant son vieux oncle vouloit se servir de l'autorité du Parlement, pour l'épouser malgré tout le monde» (VRE II, p. 8). Puis, le Président est présenté comme un être inadapté qui va à l'encontre des idéaux mondains, ignorant les règles de civilité et de la galanterie, ce que souligne clairement ce passage: "il vouloit épouser Perline malgré sa mere, et malgré ellemesme» (VRE II, p. 4). Dès lors, l'auteur en fait un jaloux de comédie qu'on peut tromper aisément et dont la conduite mène aux pires excès, suscitant la désapprobation générale.

20. Nous reprenons le concept employé par Yves Lavandier, La dramaturgie. Les mécanismes du récit [1994], Paris, Le clown et l'enfant, 2004, p. 274 et suiv.

21. Le comte de Beaujeu, cherchant à discréditer son rival aux yeux de Perline, présente en ces termes les malheurs d'une jeune femme "contrainte à passer sa vie avec un vieux mary, qui demande continuellement des soins et des complaisances, et qui juge toûjours de la vertu de sa femme, par les raisons qu'il sçait bien qu'elle a de se plaindre de luy» (VRE, p. 51-52). 
C'est effectivement ce qui se produit dans ce passage composé à la manière d'une scène de comédie. L'action se situe dans un jardin, alors que Perline fait une promenade et discute avec Dulac, qui prétend vouloir tuer Beaujeu pour la venger mais qui est, en fait, un ami du comte. Jaloux et inquiet, le Président décide d'épier sa nièce, afin de découvrir quelle est la nature des sentiments qu'elle nourrit à son égard. Il se dissimule sous un drap mouillé, ce qui accentue le ridicule du personnage, et n'entend que la partie du dialogue où Perline prétend vouloir épouser le Président. Ravi et flatté, il quitte les lieux pour ne pas être découvert et n'entend pas la fin de la conversation où Perline dévoile ses véritables intentions en affirmant «qu'elle s'étoit servie de ce pretexte pour empécher que sa mere n'engageât quelqu'un à insulter son Amant, sur la promesse de la donner pour récompense à celuy qui la vangeroit» (VRE II, p. 24-25). La théâtralité à l'œuvre dans la nouvelle de Préchac est particulièrement perceptible ici, dans la mesure où l'on retrouve l'un des poncifs de la situation théâtrale, qui consiste à " écouter et voir sans être aperçu ${ }^{22}$ ». Le Président, tel un spectateur en coulisse, regarde en cachette Perline et Dulac, ces derniers devenant à leur insu des acteurs qui échangent des répliques dans un décor digne des comédies galantes, soit les jardins d'un château. Le lecteur qui, lui, entend (lit) tout le dialogue, se divertit encore davantage, puisque le Président devient un personnage dont il peut rire, et qui non seulement se couvre de ridicule en se cachant de la sorte, mais se voit berné par son propre artifice, selon le procédé comique du trompeur trompé.

\section{La comédie des amants}

L'amour étant sans conteste le thème central du Voyage de la Reine d'Espagne, il est à l'origine de bien des aventures et donne lieu à diverses feintes ou ruses. De là, il n'y a qu'un pas à franchir pour se retrouver du côté de la comédie, ce que Préchac n’hésite pas à faire. À deux reprises, les amants vont se jouer la comédie et tenir un rôle en ayant recours au masque et au déguisement ${ }^{23}$. Fort

22. Nous reprenons ici le titre d'un article de Nobuko Akiyama, «Écouter et voir sans être aperçu: une situation théâtrale dans quelques nouvelles de $\mathrm{M}^{\mathrm{me}} \mathrm{de}$ Villedieu », dans Nathalie Grande et Edwige Keller-Rahbé (dir.), Littératures classiques, ouvr. cité, p. 191-203.

23. Les thèmes du masque et du déguisement relèvent de multiples types d'écritures. La littérature narrative du Moyen Âge est riche à cet égard, que l'on songe à la chanson de geste Gui de Nanteuil (ou Tristan de Nanteuil) ou à 
d'une tradition qui relève à la fois de la fiction narrative (contes, romans, pastorales) et du théâtre, Préchac se joue de cette double appartenance du topos du déguisement et du travestissement pour conférer une dimension dramaturgique à sa nouvelle. La première duperie met en scène Perline qui se divertit aux dépens du comte de Beaujeu. D’emblée, Préchac dédouble l'effet de théâtralité en situant l'action dans le cadre du carnaval, alors que Perline assiste à la représentation de Bellérophon, un opéra de Jean-Baptiste Lully joué pour la première fois au Palais Royal de Paris en 1679 et où l'amour est à l'honneur. Préchac évoque la beauté des lieux, du décor, de la musique et des nombreux enchantements, ce qui donne un aspect spectaculaire à l'intrigue qui se noue sous les yeux du lecteur: elle "admira les differentes decorations, et fut également charmée des belles voix, et de la symphonie [...] divertissemens qu'elle trouvoit fort à son gré» (VRE II, p. 44-45). De même, il cite deux vers de la première scène du quatrième acte de l'opéra,

Huon de Bordeaux. Selon Georges Forestier, «[1]a prose romanesque postérieure (romans et nouvelles du XIII ${ }^{e}$ au XV siècle) présentera fréquemment le thème de la poursuite amoureuse sous un travestissement, en le dépouillant de tout élément merveilleux: Le Livre du très Chevaleureux Comte d'Artois et de sa Femme, Le Roman de Pierre de Provence et la belle Maguelonne, Le Conte $d u$ Roi Flore et de la Belle Jehane (nouvelle du XIII ${ }^{\mathrm{e}}$ siècle), et une enquête approfondie en révèlerait probablement bien d'autres", (Esthétique de l'identité dans le théâtre français (1550-1680): le déguisement et ses avatars, Genève, Droz, coll. «Histoire des idées et critique littéraire», 1988, p. 445). En Italie, on retrouve ce thème entre autres dans Le Décaméron (1358) de Boccace (nouvelles II, 3 et II, 9), dans le Pecorone (1378) de Fiorentino (III, 1 et IV, 1) et dans le Novellino (1476) de Salernitano. Quant aux auteurs de la fin du Moyen Âge et du début de la Renaissance, ils l'emploient davantage comme ressort dramatique, notamment dans La Calandria (1513) de Bibbiena et dans Gl'Ingannati (1531) de l'Académie siennoise des Intronati. Toujours selon Georges Forestier: "Si les comédies italiennes postérieures sont nombreuses à présenter des jeunes filles travesties, exerçant ainsi une influence directe sur le théâtre français à travers les adaptations de Larivey, il faut leur adjoindre le roman pastoral de Montemayor, Diana (1559), qui laissera une empreinte profonde sur une bonne part de la production littéraire (narrative et dramatique) européenne, particulièrement avec l'épisode de Felismena, dont on peut trouver l'influence diffuse dans L'Astrée et directe dans Félismène de Hardy» (Esthétique de l'identité dans le théâtre français (1550-1680), ouvr. cité, p. 446-447). Pour sa part, Jean Rousset remarque que de nombreux auteurs dramatiques, tels Rotrou, Mareschal, Scudéry, Pichou, Mairet, Corneille, se plaisent à construire des intrigues qui reposent essentiellement sur les thèmes du déguisement et du trompe-l'œil, de sorte que «[t]out le monde est masqué; personne ne se reconnaît plus; chacun passe pour autre qu'il n'est, personne n'est ce qu'il paraît» (Jean Rousset, La littérature à l'âge baroque en France. Circé et le paon [1954], Paris, J. Corti, 1968, p. 54). 
qui correspondent à l'état d'âme de Perline: "Quand on obtient ce qu'on aime,/Qu'importe, qu'importe à quel prix» (VRE II, p. 47); et décrit ainsi la réaction de la jeune femme: "Cét air, ou peut estre ces paroles, se trouverent si conforment à ses pensées, qu'elle ne pût s'empescher de se recrier, et d'aplaudir à la personne qui chantoit, il luy échapa ensuite quelques soûpirs» (VRE II, p. 47). Par ce jeu de renvoi intertextuel, Préchac semble vouloir inscrire cette scène dans la tradition dramatique et inciter son lecteur à la lire comme une comédie, en adoptant le regard du spectateur.

L'auteur accumule, dans un effet de surenchère, les éléments qui accentuent la théâtralité de la scène, préparant le lecteur à assister à la comédie qui va se dérouler. D’abord, il plante le décor en précisant que Perline prend place «dans une loge grillée où personne ne la verroit» (VRE II, p. 42), qu'elle s'y rend masquée pour cacher son identité et qu'elle se retrouve en présence de «deux personnes de qualité, qui ne vouloient point estre connuës» (VRE II, p. 43-44), dont « un homme enveloppé dans un manteau» (VRE II, p. 44), ce qui confère une atmosphère de mystère propice aux intrigues. Le recours au déguisement n'est pas fortuit, puisque ce procédé permet de «mettre en lumière le comique à double détente de la situation ${ }^{24}$ », et qu'il donne lieu à une série de méprises et de rebondissements. Transportés par les airs d'opéra, Perline et l'inconnu se découvrent un instant, mais l'obscurité est telle que l'homme ne peut qu'être saisi par la beauté de la jeune femme, alors que Perline a le temps de reconnaître, non sans surprise, qu'il s'agit du comte de Beaujeu. Perline profite de son avantage pour mettre à l'épreuve la fidélité de son amant et se divertir à ses dépens; jouant la belle inconnue, elle se cache derrière son masque et déguise sa voix. On retrouve là la forme comique du quiproquo où un personnage prend une personne pour une autre, méprise dont le lecteur devient complice et qui provoque le rire. Notons que la scène a été soigneusement préparée par Préchac qui précise que Beaujeu ne peut s'attendre à trouver Perline à Paris, puisque, s'imaginant qu'elle fera le voyage depuis Bordeaux «par petites journées, il luy manda qu'elle pourroit estre à Paris dans trois semaines» (VRE II, p. 38-39). Or, Perline arrive « huit jours plûtost» (VRE II, p. 40), ce qui rend la méprise d'autant plus vraisemblable et permet à Préchac d'intégrer un dialogue à double entente entre les deux amants:

24. Nous reprenons ici la définition proposée par Philippe Bousquet, Agnès Carbonelle et Claudine Castel, La comédie et le comique, Paris, Nathan, 2000, p. 80 . 
on ne me regarde pas impunément, répliqua Perline, je suis asseurée que vous m'aimerez aussi-tost que vous m'aurez veuë; ainsi, avant que de vous exposer à ce péril, voyez si vostre constance pourra s'accomoder d'une nouvelle passion? Voilà de grandes menaces, dit le Comte, mais sçavez-vous qu'il n'est pas si aisé que vous pensez de rendre infidèle un homme qui aime bien (VRE II, p. 54-56).

Connaissant la véritable identité de Perline, alors que le comte de Beaujeu l'ignore, le lecteur, à l'instar de Perline, peut rire des quiproquos qui s'ensuivent. Son statut de spectateur privilégié rend donc l'effet comique assuré.

La seconde duperie des amants prend la forme d'une comédie savamment orchestrée qui s'insère au sein d'une autre comédie. En effet, Perline, voulant sauver la vie du comte qui est menacé d'exécution pour s'être battu en duel contre Pontignac, se retire dans un couvent. Profitant des altérations qui paraissent sur son corps et modifient sa physionomie, "elle s'avisa enfin de feindre qu'elle estoit malade, ce qu'elle n'eut pas de peine à persuader à cause du grand changement qu'on remarquoit sur son visage» (VRE II, p. 101-102). Prétextant être mourante, elle fait venir un confesseur à son chevet et lui avoue que les preuves données contre Beaujeu pour gagner son procès sont fausses. La stratégie fonctionne, puisque les charges retenues contre Beaujeu tombent et qu'il obtient aussitôt sa remise en liberté. Cette imposture sert en quelque sorte de récit-cadre à la comédie qui suit et qui retiendra plus particulièrement notre attention. Le comte de Beaujeu, cherchant un expédient pour voir sa maîtresse, décide de prendre l'identité d'un faux prieur et d'accompagner le Président qui se rend assidûment auprès de sa nièce, qu'il espère toujours épouser. La théâtralité intervient ici, puisque le lecteur assiste, comme s'il était en coulisse, aux préparatifs du comte qui se déguise pour mieux tenir son rôle: «il prit une fausse barbe [...] après s'estre habillé d'un habit convenable à ce qu'il vouloit paroistre » (VRE II, p. 110-111). Ayant dupé le Président aussi bien que les religieuses par ce déguisement, le comte obtient un entretien particulier avec Perline, auquel le lecteur assiste avec amusement. Reprenant le procédé comique de la méprise sur l'identité, Préchac insère à nouveau un dialogue à double entente entre les amants, où le faux prieur (Beaujeu) recommande à Perline de se guérir de son mystérieux mal en voyant celui qu'elle aime. L'homme en profite au passage pour l'assurer de la fidélité et de la sincérité de l'amour de Beaujeu à son égard, propos qui surprennent grandement Perline. 
Cependant, la scène de reconnaissance, dont le lecteur et le comte attendent une issue heureuse, connaît un revirement de situation. Voulant mettre un terme à ce divertissement, Beaujeu retire sa fausse barbe, non sans avoir volontairement ménagé un effet de surprise. Ce manège donne lieu à un tableau où Préchac accorde une large part à la description d'éléments visuels, afin que le lecteur puisse se représenter parfaitement la scène: "tournez un moment la teste, et vous verrez un effet merveilleux de mes secrets. Perline luy obeït, et dans cét instant le Comte osta sa barbe, et se mit à genoux au pied du lit de sa Maîtresse, qui s'esta[i]t tournée pour voir cette merveille» (VRE II, p. 119). Or le comte est victime de son talent, puisque Perline, ne l'ayant pas reconnu sous son déguisement, est si étonnée que sa réaction trahit la présence de Beaujeu au couvent: "[elle] fut si surprise de ne trouver plus le Prieur et de remarquer à sa place un visage semblable à celuy de son Amant, qu'elle fit un cry qui épouvanta les Religeuses " (VRE II, p. 120-121). Préchac, avec un souci de la mise en scène évident, donne ce que l'on peut considérer comme une série d'indications scéniques, précisant la gestuelle et les déplacements des personnages, en particulier ceux du comte, contraint à fuir, ce qui permet d'assister à la scène comme au théâtre:

il n'eût jamais l'adresse de remettre sa barbe, et s'enfuit la barbe à sa main au travers des Religieuses, qui se regardoient fort étonnées; il arriva à la porte ou les clefs se trouverent heureusement pour luy, mais il estoit si troublé qu'il ne sceut jamais profiter de ce bonheur [...], il traversa un petit corridor, et entra dans une chambre qui par hazard se trouva ouverte, il poussa la porte assez doucement, et s'enferma dedans (VRE II, p. 120122).

Ce premier acte semble être une répétition générale annonçant le second acte à venir. En effet, Beaujeu, enfermé au couvent, tente à nouveau de voir Perline plutôt que de se sauver et, cette fois encore, il a recours au déguisement. Il se travestit ${ }^{25}$ alors en

25. Ainsi que le fait remarquer Sylvie Steinberg, nombreux sont les auteurs qui ont exploité le thème du travestissement sous l'Ancien Régime: «Le thème du travesti n'est pas réservé à la littérature pornographique ou licencieuse. Dans la littérature de l'époque moderne, la conquête amoureuse en habit travesti est un cliché rebattu. Combien de héros déguisés qui séduisent des êtres du même sexe sous une fausse apparence? Combien de héros qui trompent des êtres du sexe opposé en les plongeant dans le trouble? Des jumeaux de $L a$ Nuit des rois aux héroünes de Marivaux, les personnages qui se travestissent pour séduire recourent à toutes sortes de stratagèmes ", Sylvie Steinberg, 
religieuse tout en prenant soin de déguiser sa voix ${ }^{26}$. Le succès du travestissement lui permet de se rendre auprès de Perline mais, craignant qu'elle ne soit trompée par l'illusion, il lui écrit un billet pour la préparer à sa venue, lui mandant de «ne gastez rien par [son] étonnement» (VRE II, p. 133). Les amants discutent donc librement, s'amusant de cette surprenante aventure qui se déroule à l'insu des religieuses et du Président, que Perline fuit en se cachant au couvent.

Le troisième acte de cette comédie des amants, où les péripéties et les obstacles s'accumulent, a pour moteur une règle de bienséance. La vertu et la pudeur de Perline ne l'autorisant pas à passer la nuit avec Beaujeu, elle lui demande de se retirer. Le comte se cache dans la chambre d'une religieuse morte depuis peu et qui a menacé de revenir hanter le couvent. Déjà, le lecteur voit se mettre en place les éléments du spectacle terrifiant qui va se dérouler sous ses yeux et, surtout, sous ceux des religieuses. En effet, une nonne, apercevant de la lumière filtrer par la serrure de la porte de cette chambre, croit voir le spectre de la religieuse décédée et elle en fait part à une consœur qui la traite de visionnaire. Les deux femmes se rendent à la chambre et, cette fois, Beaujeu tire parti de la situation. Il laisse les religieuses se méprendre sur son identité, devenant alors acteur mais aussi spectateur de la comédie qu'il va jouer à leur insu. Il tient donc le rôle de la revenante, en ajustant son déguisement et sa voix pour mieux entretenir l'illusion. Préchac insiste particulièrement sur la réaction des religieuses effrayées, spectatrices de cette comédie qu'elles croient véritable: «[Beaujeu] ne laissa pas de leur parler d'un ton de voix extraordinaire, qui épouvanta tellement les timides Religieuses, qu'elles s'enfuirent en criant au secours de toute leur force, toute la Communauté fut allarmée en un instant» (VRE II, p. 143-144). Il insère également un dialogue en style direct, qui se déroule de part et d'autre de la porte de la chambre et au cours duquel les religieuses écoutent avec crainte le supposé fantôme qui leur recommande de protéger Perline de son oncle mal intentionné. La confusion générale qui s'ensuit est favorable au comte de Beaujeu, puisqu'elle lui permet de s'enfuir du couvent, mais ce n'est pas sans avoir troqué son habit de religieuse pour celui de faux prieur. On revient alors à la

La confusion des sexes. Le travestissement de la Renaissance à la Révolution, Paris, Fayard, 2001, p. 38-39.

26. Voir la description détaillée du comte enfilant l'habit de religieuse et prenant soin d'ajuster son voile dans VRE II, p. 129-131. 
première comédie qui sert de récit-cadre à celle des amants, où le Président tient le rôle du personnage berné par cette duperie qui respecte la règle des 24 heures: «Le Président [...] ne comprenoit plus rien à tout ce qu'il voyoit, et moins encore à ce qui luy estoit arrivé pendant la journée» (VRE II, p. 151). On le voit, Préchac intègre habilement de la théâtralité à sa nouvelle. Par une série d'indications scéniques et visuelles qui précisent les déplacements, le ton de la voix et l'habillement de ses personnages, l'auteur prépare son lecteur à rire de la comédie que les amants vont jouer. Il élabore minutieusement cette comédie en trois actes, où le masque, le déguisement et le travestissement, par les imbroglios qu'ils entraînent, agissent comme un ressort comique des plus efficaces.

Si René Godenne affirme que Préchac est un «[a]uteur sans originalité, sans grands talents aussi (rien de plus impersonnel que son style par exemple), [...] se content[ant] de reprendre des schémas d'intrigue traditionnels, où se retrouvent tous les poncifs romanesques du temps ${ }^{27}$ », il nous semble plutôt que Préchac est un auteur qui a largement contribué au succès du genre de la nouvelle en France par ses diverses expérimentations, brouillant ainsi les délimitations de ce genre polymorphe aux contours flous. Les emprunts au genre dramatique semblent avoir joué un rôle prépondérant dans sa pratique d'écriture, et notamment dans le cas du Voyage de la Reine d'Espagne. D'abord, le sujet de l'intrigue doit beaucoup à la tragicomédie du Cid de Corneille, mais est nettement infléchi du côté de la comédie galante. Ensuite, on retrouve les personnages et les situations traditionnels de la comédie, tels la vieille coquette et le rival incommode qui compromettent le mariage heureux entre les parfaits amants, ainsi que les procédés comiques d'usage, dont le quiproquo, la méprise sur l'identité ou encore le trompeur trompé, éléments qui instillent une forme d'ironie dramatique dans la nouvelle. Enfin, les mentions relatives aux jeux scéniques, à la présence des personnages sur scène, à leurs déplacements, à leur expression et à leur voix, ainsi que l'ajout de dialogues participent à cette théâtralité. Si l'auteur insère les scènes dramatiques afin de ponctuer les temps forts de son récit, il tire

27. René Godenne, «Préface», dans Jean de Préchac, L'Illustre Parisienne. Histoire galante et véritable [1678-1690], Genève, Slatkine Reprints, 1980, p. xi. 
également profit du dynamisme de la composition théâtrale qui lui permet de garder le lecteur en haleine, selon le principe de l'action continue, l'entraînant ainsi, comme au théâtre, de surprises en étonnements. On serait tenté de conclure que si Préchac combine les procédés de l'écriture dramatique et romanesque, c'est dans la mesure où il cherche à joindre un large public, qu'il soit lecteur de nouvelles ou spectateur de théâtre. Déterminé à plaire à ses lecteurs et à les divertir, il doit sans cesse se renouveler et n'hésite donc pas à expérimenter diverses pratiques d'écriture. Dans ce contexte, le recours à la théâtralité serait pour lui une stratégie destinée à renouveler la forme d'un genre qui est au goût du jour et à amuser un public friand de nouveauté. 